\title{
Microporous Nano-hydroxyapatite/collagen/phosphatidylserine Scaffolds Embedding Collagen Microparticles for Controlled Drug Delivery in Bone Tissue Engineering
}

\author{
Chunrong Yang ${ }^{a, b} *$ Cong Fang ${ }^{a, b}$ \\ ${ }^{a}$ School of Materials Science and Engineering, Fujian University of Technology - FJUT, \\ Fuzhou 350118, China \\ ${ }^{b}$ Fujian Provincial Key Laboratory of Advanced Materials Processing and Application, \\ Fuzhou 350118, China
}

Received: July 22, 2015; Revised: September 7, 2015

\begin{abstract}
Scaffolds featuring spatiotemporal control of drug release is highly desirable for bone tissue regeneration. The objective of this study was to construct a scaffold with gradient porosity and drug distribution and evaluate the effect of scaffold structure on drug release kinetics and cell bioactivity. Nano-hydroxyapatite/collagen/phosphatidylserine scaffolds embedded with steroidal saponin loaded collagen microparticles were prepared using a porogen leaching protocol. Morphological characterization showed that the scaffolds consisted of dense layer and loose layer, and pores were interconnective. The microparticles were entrapped at the center of the scaffolds follow a gradient distribution. Release kinetics correlated with the structure. The loose layer showed greater drug release amount as compared to the dense layer. Such differences in release kinetics have distinct effects on cell bioactivity. Cell proliferated much more in loose layer than that in the dense layer. Such spatial and temporal control over drug deposition and delivery within the scaffolds could provide opportunities for tissue regeneration associated with optimum drug doses at wound site, and lessen undesirable drug release and side-effects at uninjured site.
\end{abstract}

Keywords: scaffold, microparticle, controlled release, bone

\section{Introduction}

Drug loaded delivery system is considered an emerging alternative for the regeneration of bone defects ${ }^{1}$. Such drug provide readily available bioactivity facilitating bone ingrowth, Steroidal saponins (SS) has been demonstrated to promote the proliferation of osteoblasts. Moreover, the biomedical potential of SS loaded biodegradable collagen microparticles as a drug delivery system has been demonstrated in our previous study ${ }^{2}$. For bone repair, an ideal delivery system should combine an osteoconductive scaffold as structural support for cells with a drug for cellular growth and differentiation ${ }^{3,4}$.

The challenge of such scaffolds to mimic physiological patterns lies in (i) an optional control of independent drug release kinetics of more than one direction. (ii) control of appropriate release rates upon tissue. (iii) the potentially targeted drug delivery to specific cells, and (iv) the preservation of drug functionality and integrity during construction of the scaffold ${ }^{5,6}$.

So far little studies reported how the spatiotemporal control of drug may affect tissue repair. The principal significance has been described for spatially separated release and deposition of drug, showing an interesting correlation between drug pharmacokinetics and material structure ${ }^{7}$. Moreover, manufacturing processes of scaffolds with spatiotemporal control of drug in bone repair are challenging.

*e-mail:lambmeo@163.com
The extracellular matrices of hard tissue are composed of organic and inorganic phases. The inorganic phase consists primarily of hydroxyapatite (HA), whilst the organic phase consists mainly of type I collagen (COL1) and small amounts of ground substance, such as glycosaminoglycans, proteoglycans, glycoproteins and phosphatidylserine (PS), all with unique distributions reflecting their biological roles. HA, COL and PS, when processed into biomaterial, results a bone scaffold with appropriate bio-degradation, excellent mechanical properties, and well bioactivity and biocompatibility, which has been demonstrated in our previous work ${ }^{8}$. Here we introduce a protocol to fabricate inhomogeneous HA/COL/PS (HCP) scaffolds with gradient distribution of SS loaded collagen microparticles. We discussed in detail the impact of scaffold structure on release kinetics of drug to estimate the spatiotemporal control of drug delivery. Cellular response was tested with MC3T3-E1 cells to assess the impact of drug release on the proliferation and migration of cells when seeded onto different surface of the scaffolds.

\section{Material and Methods}

\subsection{Preparation of paraffin spheres}

Paraffin spheres were used as porogen for the preparation of HCP scaffolds. They were prepared as described before? Briefly, gelatin (Sigma-Aldrich Co., Saint Louis, USA) was dissolved at $80{ }^{\circ} \mathrm{C}$ in ultrapure water at a concentration 
of $30 \mathrm{~g} / 1$ and mixed with paraffin (Yudao Co., Chengdu, China) upon melting at a final concentration of $200 \mathrm{~g} / \mathrm{l}$. Phases was emulsified for $20 \mathrm{~min}$ and then poured into ice water. The solidified paraffin spheres were collected and washed with ultrapure water. After drying, the paraffin spheres with the diameter of 180-250 $\mu \mathrm{m}$ were collected with standard sieves.

\subsection{Preparation of HCP scaffolds}

SS loaded collagen microparticles were prepared in previously research and the particle size of microparticles ranged from several microns up to about $100 \mu \mathrm{m}$.

HCP scaffolds were prepared as follows. In brief, paraffin spheres of 180-250 $\mu \mathrm{m}$ diameter were filled into $20 \mathrm{ml}$ cylindrical syringe, followed by sintered at $37^{\circ} \mathrm{C}$ for $40 \mathrm{~min}$ in order to achieve sufficiently interconnected pores within the scaffold. After the heat treatment the syringe was annealed to room temperature.

HCP scaffolds embedding collagen microparticles were prepared by mixing previously fabricated collagen microparticles with the paraffin spheres prior to heating the spheres at $37^{\circ} \mathrm{C}$. After annealing to room temperature, nano-hydroxyapatite, phosphatidylserine, and collagen solution were added to the syringe and carefully aspirated to fill the interspace between the spheres. The constructs were in multiple layers and the pores were interconnective and gradient, which can be controlled by gradient distribution of drug loaded collagen microparticles and paraffin spheres (Figure 1).

The preparations were then shock-frozen in liquid nitrogen, and freeze dried at $-30{ }^{\circ} \mathrm{C}$ for $45 \mathrm{~h}$ (ALPHA2-4, Christ, Germany). Scaffolds were treated with $90 \%(\mathrm{v} / \mathrm{v})$ methanol solution for $45 \mathrm{~min}$ and then treated with hexane for $20 \mathrm{~h}$ to extract the paraffin spheres. Hexane was replaced after $24 \mathrm{~h}$ and the resulting scaffolds were dried under vacuum for $10 \mathrm{~h}$ (YB-FD-1, Yibei Co., Shanghai, China) in order to completely dry the scaffolds and minimize residual hexane ${ }^{10}$.

\subsection{Cross-section observation of HCP scaffolds}

The cross-section of HCP scaffolds with embedded collagen microparticles was coated with gold and morphologically determined using scanning electron microscopy (SEM) (S-3400N, Hitachi Co., Tokyo, Japan).

\subsection{In vitro drug release}

The temporal and spatial release experiment of the HCP scaffolds was performed in a continuous flow system (Figure 2). The in vitro simulated body fluid employed for the release medium was prepared by dissolving reagent-grade $\mathrm{NaCl}$ $\left(142 \mathrm{mmol} \bullet 1^{-1}\right), \mathrm{NaHCO}_{3}\left(4.2 \mathrm{mmol} \cdot \mathrm{l}^{-1}\right), \mathrm{KCl}\left(3.0 \mathrm{mmol}^{-1} \mathrm{l}^{-1}\right)$, $\mathrm{K}_{2} \mathrm{HPO}_{4} \cdot 3 \mathrm{H}_{2} \mathrm{O}\left(1 \mathrm{mmol} \cdot \mathrm{l}^{-1}\right), \mathrm{CaCl}_{2}\left(2.6 \mathrm{mmol}^{-1} \mathrm{l}^{-1}\right), \mathrm{Na}_{2} \mathrm{SO}_{4}$ $\left(0.5 \mathrm{mmol}^{-1} \mathrm{l}^{-1}\right)$, and $\mathrm{MgCl}_{2} \cdot 6 \mathrm{H}_{2} \mathrm{O}\left(1.6 \mathrm{mmol} \cdot \mathrm{1}^{-1}\right)$ in deionized water. The solution was buffered at PH 7.4 with trishydroxymethylaminomethane $\left[\left(\mathrm{CH}_{2} \mathrm{OH}\right)_{3} \mathrm{CNH}_{2}\right]$ and $1 \mathrm{~N} \mathrm{HCL}$ at $37^{\circ} \mathrm{C}$.

To assess the effect of the structure of the scaffolds on the release kinetics of drug, the samples were laid down the filter at $37^{\circ} \mathrm{C}$ with the loose surface as the input side and the dense surface as the output side, or reversed (Figure 2). After specific time periods, the outflow solution was collected, and the inflow solution was refreshed. The steroidal saponin content of solution collected for each interval was determined by using the ultra performance liquid chromatography (UPLC, Waters Co., Milford, USA), and steroidal saponins were used as the standard.

\subsection{In vitro cell bioactivity on HCP scaffolds}

The osteoblast bioactivity of the HCP scaffolds in bone formation was investigated using MC3T3-E1 cells (Japan Riken Cell Collection). Cells were cultured in the DMEM-10\% FBS medium (Gibco), and maintained in $5 \% \mathrm{CO}_{2}$ at $37^{\circ} \mathrm{C}$. The cells $\left(2 \times 10^{3}\right.$ cells $\left./ \mathrm{mL}\right)$ were seeded on the dense or loose surface of $30 \mathrm{mg}$ of specimens, placed on the bottom of $100 \mathrm{~mm}$ plastic tissue-culture dishes (Corning Co.,Midland, USA) and remained undisturbed in a humidified incubator $\left(37^{\circ} \mathrm{C}\right.$ and $\left.5 \% \mathrm{CO}_{2}\right)$.

The morphology of cells grown on different surfaces of the scaffolds was observed using a confocal laser scanning microscope (CLSM, TCS SP5, Leica, Wetzlar, Germany). After $5 \mathrm{~h}$ of incubation, the specimens with cells were fixed by $4 \%$ paraformaldehyde in PBS at room temperature, followed by wash. They were then immersed in $1 \%$ rhodamine phalloidin for $10 \mathrm{~min}$ to stain cell. After rinsing with phosphate buffered saline (PBS), the stained specimens were placed on the glass-bottomed dish and examined, then fluorescence density of the cell was measured.

The growth kinetics of MC3T3-E1 cells on both surfaces of the scaffolds were assessed using a 3-(4,5-dimethyl2-thiazolyl)-2,5-diphenyl-2H tetrazolium bromide (MTT) (Sigma Co., Saint Louis, USA) assay. The cells were cultured for 1,3 , or 5 days, respectivly. Subsequently, the medium was removed and $200 \mu \mathrm{l}$ MTT solution was added to each well for incubation at $37^{\circ} \mathrm{C}$ for $4 \mathrm{~h}$. Then the MTT solution was removed and the insoluble blue formazan crystal was dissolved in dimethyl sulfoxide (DMSO) (Sigma Co., Saint Louis, USA). Subsequently, optical density of the solution was measured. The absorbance was directly proportional to cell proliferation.
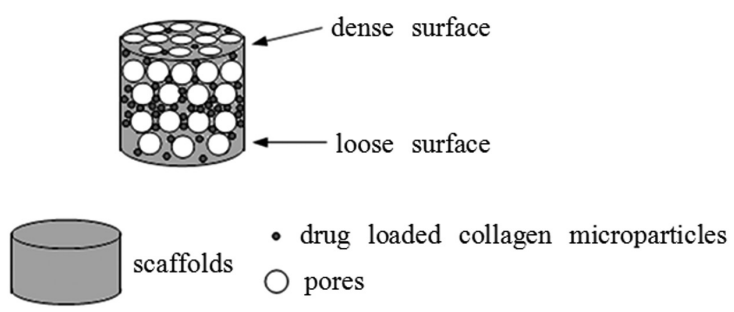

Figure 1. Illustration showing gradient distribution of drug loaded collagen microparticles in HCP scaffolds.

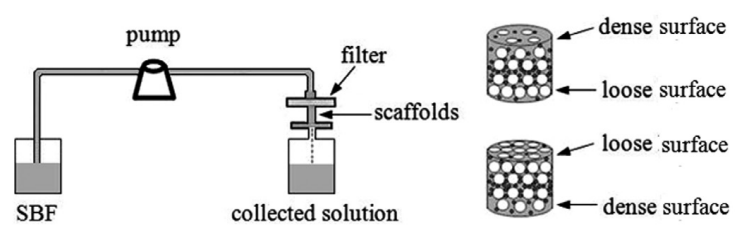

Figure 2. Continuous flow system. 


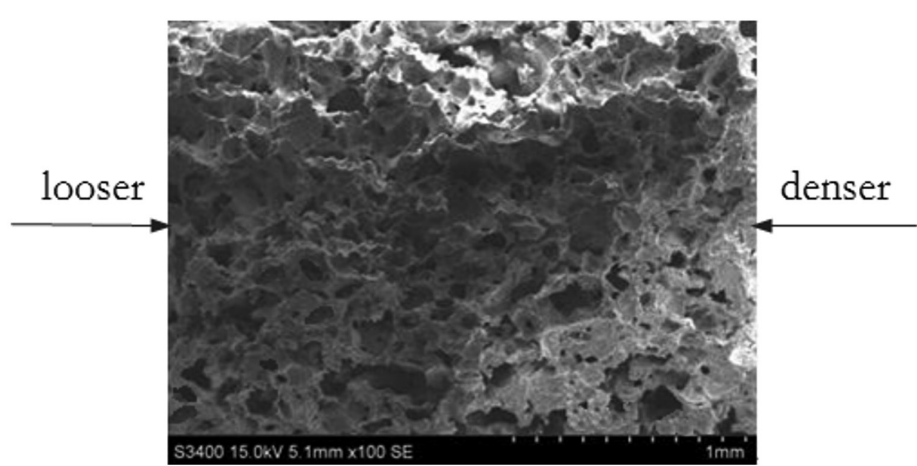

(a)

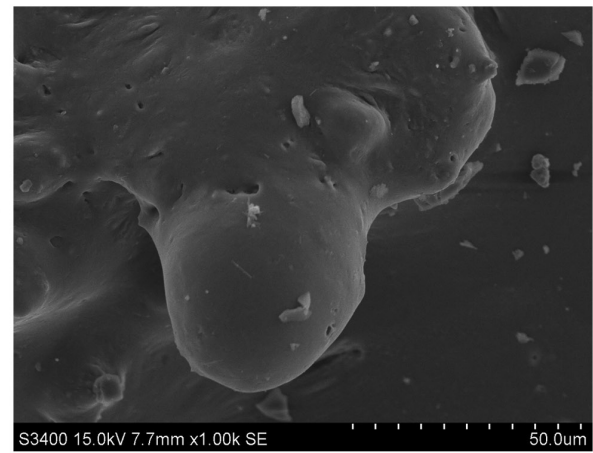

(b)

Figure 3. SEM images of the cross-section of the HCP scaffolds (a) and SS loaded collagen microparticles embedded in the scaffolds (b).

\subsection{Statistical analysis}

The data were reported as the mean $\pm \mathrm{SD}$. Two-factor analyses of variance (ANOVAs) with Bonferroni-Dunn and Fisher's post hoc tests for multiple comparisons as well as Student's t test were performed using Sigma StatTM Software for Windows Version 2.03, SPSS Inc. Significance was accepted at a level of $\mathrm{P}<0.05$.

\section{Results and Discussion}

\subsection{Morphological characterization of HCP scaffolds}

SEM observation of the HCP scaffolds showed a continuous and gradient structure of irregular interconnected pores, and consisted of dense layer and loose layer (Figure 3a). Most of the pore sizes were around 100-300 $\mu \mathrm{m}$. Paraffin leaching from SS loaded HCP matrices with hexane resulted in porous structure of high interconnectivity. Also matrix between the interconnecting pores was observed in this case. The embedded collagen microparticles to be well integrated into the HCP scaffolds, strictly locating in the intersections or walls of the matrices (Figure 3b). Also embedded collagen microparticles appeared to be coated with a thin layer of HCP matrices.

Such three-dimensional gradient structure may vary release profile and enhance the efficiency of the drug in bone regeneration by creating and maintaining appropriate space for progenitor cell migration, proliferation, and differentiation.

\subsection{In vitro drug release studies}

In vitro SS release kinetics of collagen microparticles embedded in HCP scaffolds, and the effect of the structure of the scaffolds on the release kinetics were evaluated for 30 days in Figure 4. Irrespective of the flow direction, the release profiles were triphasic consisting of (i) a typical burst release within the first $12 \mathrm{~h}$, followed by (ii) a period of low release rates until day 7 (the dense surface to loose surface) and day 12 (the loose surface to dense surface), and finally (iii) a marked increase in release from these days on.

The drug from the dense surface to loose surface of the scaffolds showed a burst release of $25.8 \pm 3.8 \%$ after $0.5 \mathrm{~d}$.

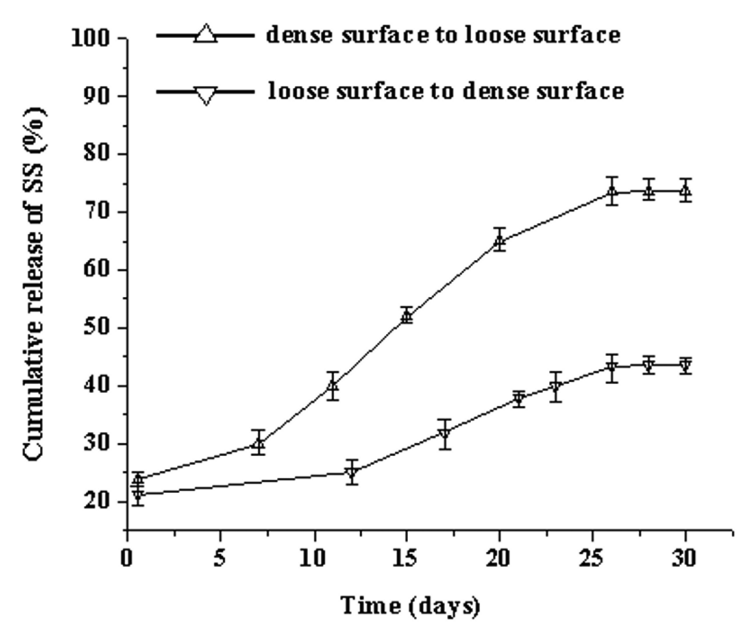

Figure 4. Cumulative release of SS from HCP scaffolds embedded with SS loaded collagen microparticles when SBF flowed along different directions.

The burst release from the loose surface to dense surface was less prominent with $21.3 \pm 4.7 \%$. These findings were further reflected by the release rate throughout the observation period. The average release rate of drug from the dense surface to loose surface exceeded significantly $(p<0.05)$ the rate from the loose surface to dense surface. Almost no drug was released from scaffolds until day 26 , turning into a continuous and slow release from then on. After 30 days, $73.7 \pm 4.0 \%$ of the initially loaded drug was released from the dense surface to loose surface of the scaffolds. On the other hand, much less, only $43.6 \pm 3.1 \%$ of the initially loaded drug was released from the loose surface to dense surface of the scaffolds.

The difference of release profiles of drug from scaffolds has been largely affected by the flow direction. This outcome was explained to result from the diffusional barrier through the scaffold matrix. From the loose surface to dense surface, drug diffusion was driven from low to high resistance, which induced slow release. In contrast, from the dense surface to loose surface diffusion was faster. 
Such differences in release kinetics of drug from scaffolds with different exposed surfaces may have distinct effects on bone regeneration ${ }^{7}$. In this study, we could show that the release profiles were spatiotemporal. This may be an

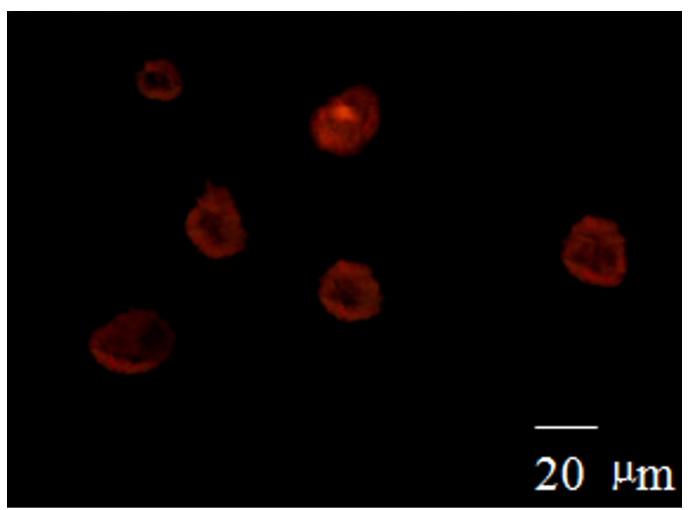

(a)

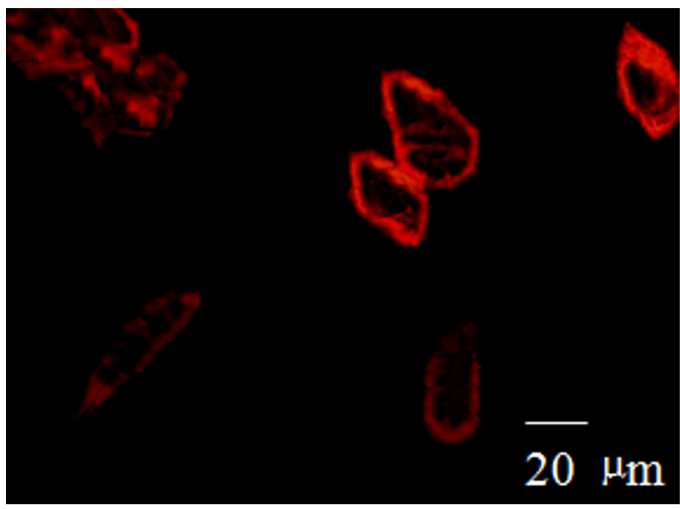

(b)

Figure 5. CLSM micrographs of MC3T3-E1 cells adhering to the different surfaces of the HCP scaffolds embedded with SS loaded collagen microspheres after $5 \mathrm{~h}$ of inoculation: (a) cells seeded on the loose surface of the scaffolds. (b) cells seeded on the dense surface of the scaffolds.

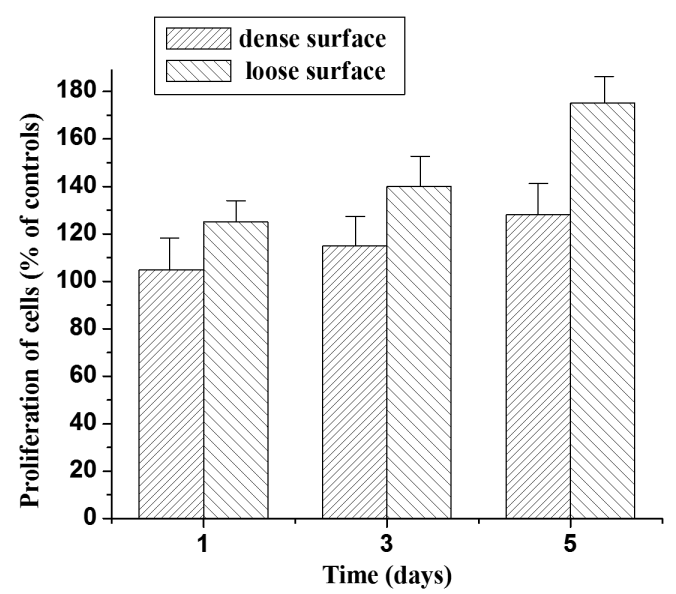

Figure 6. Proliferation of MC3T3-E1 cells on both surfaces of the HCP scaffolds. At the indicated time points, cell viability of the loose surface group was significantly higher than those of the dense surface group. Data are presented as means \pm standard deviations for $n=5$. advantage as compared to homogeneous structure of scaffolds. This would direct the drug much into the loose layer of the scaffold from where they may be easily washed out and exposed to the defect tissue. Additionally, the smaller releas amount in another surface when exposed to well-surrounding site could avoid potential toxicity and reduce undesirable side-effects, such as hyperplasia, hypertrophy, metaplasia, ectopic bone formation, or unwanted vessel growth ${ }^{11}$. This leads to the conclusion that the constructed inhomogeneous scaffolds incorporating drug-loaded microparticles could allow spatially separated release of drug, a prerequisite for the regeneration of bone defects.

\subsection{Cell bioactivity}

A MC3T3-E1 cell proliferation assay was used to assess if the bioactivity of released drug was impacted by the inhomogeneous structure of scaffolds. According to the fluorescence intensity (Figure 5), it could be found that cell grown on the loose surface of the scaffolds spread fully, interconnected or formed dense cell clusters in the scaffold matrix after $5 \mathrm{~h}$ of culture. On the other hand, most cells grown on dense surface of the scaffolds were round or oval, and area of cell spreading was smaller.

This result was further confirmed by the cell-proliferation (Figure 6). Compared to the loose surface, cell grown on the dense surface of the scaffolds induced significantly lower proliferation within the same culture time.

This outcome could be explained that released drug in combination with different surfaces of the scaffolds had substantial effects on the cell growth and proliferation. We previously demonstrated the SS on the bioactivity of MC3T3-E1 cells. The growth and proliferation of cells related to the release of SS from collagen microparticles. From above release experiments, we would have expected high proliferation rates on the loose layer of the scaffolds when considering the high release of drug. The HCP scaffolds may possibly act similar to the extracellular matrix, and provide a depot for drug that is released surface-bound to stimulate different cell growth rates. Therefore controlled release of drug could deliver optimum dose for the wound tissue to induce cell growth and promote tissue regeneration, while lessening the undesired release to uninjured areas to avoid side effects.

\section{Conclusions}

We developed an inhomogeneous and gradient HCP scaffold embedding collagen microparticles which we prior loaded with SS. Different surface of scaffold resulted in distinct drug release kinetics and cell population. Spatial control of drug deposition and temporal control of drug delivery within the scaffolds could provide opportunities for tissue regeneration associated with optimum drug doses at wound site, and reduce undesirable drug release and side-effects at uninjured site. Future, pre-clinical studies will be needed to investigate the performance of SS loaded scaffolds on bone in vivo.

\section{Acknowledgements}

This work was financially supported by natural science foundation project of Fujian (Grant no. 2014J05054) and scientific research starting foundation project of Fujian university of technology (Grant no. GY-Z0854). 


\section{References}

1. Nagai N, Kumasaka N, Kawashima T, Kaji H, Nishizawa M and Abe T. Preparation and characterization of collagen microspheres for sustained release of VEGF. Journal of Materials Science. Materials in Medicine. 2010; 21(6):1891-1898. http://dx.doi. org/10.1007/s10856-010-4054-0. PMid:20232232.

2. Yang $\mathrm{C}$ and Wang J. Preparation and characterization of collagen microspheres for sustained release of steroidal saponins. Materials Research. 2014; 17(6):1644-1650. http://dx.doi. org/10.1590/1516-1439.307214.

3. Chen J, Wang Z, Wen Z, Yang S, Wang J and Zhang Q. Controllable self-assembly of mesoporous hydroxyapatite. Colloids and Surfaces. B, Biointerfaces. 2015; 127:47-53. http:// dx.doi.org/10.1016/j.colsurfb.2014.12.055. PMid:25638722.

4. Chen J, Yu Q, Zhang G, Yang S, Wu J and Zhang Q. Preparation and biocompatibility of nanohybrid scaffolds by in situ homogeneous formation of nanohydroxyapatite from biopolymer polyelectrolyte complex for bone repair applications. Colloids and Surfaces. B, Biointerfaces. 2012; 93:100-107. http://dx.doi. org/10.1016/j.colsurfb.2011.12.022. PMid:22297206.

5. Li F, Wu W, Xiang L, Weng G, Hong H, Jiang H, et al. Sustained release of VH and rhBMP-2 from nanoporous magnesium-zincsilicon xerogels for osteomyelitis treatment and bone repair. International Journal of Nanomedicine. 2015; 22(10):40714080. http://dx.doi.org/10.2147/IJN.S82486. PMid:26124660.

6. Boukari Y, Scurr DJ, Qutachi O, Morris AP, Doughty SW, Rahman CV, et al. Physicomechanical properties of sintered scaffolds formed from porous and protein-loaded poly(DLlactic-co-glycolic acid) microspheres for potential use in bone tissue engineering. Journal of Biomaterials Science. Polymer Edition. 2015; 26(12):796-811. http://dx.doi.org/10.1080/092 05063.2015.1058696. PMid:26065672.

7. Li X, Wang J, Su G, Zhou Z, Shi J, Liu L, et al. Spatiotemporal control over growth factor delivery from collagen-based membrane. Journal of Biomedical Materials Research. Part A. 2012; 100(2):396-405. http://dx.doi.org/10.1002/jbm.a.33282. PMid:22083844.

8. Yang C, Wang Y and Chen X. Preparation and evaluation of biomimetric nano-hydroxyapatite-based composite scaffolds for bone-tissue engineering. Chinese Science Bulletin. 2012; 57(21):2787-2792. http://dx.doi.org/10.1007/s11434-012-52014.

9. Wenk E, Meinel AJ, Wildy S, Merkle HP and Meinel L. Microporous silk fibroin scaffolds embedding PLGA microparticles for controlled growth factor delivery in tissue engineering. Biomaterials. 2009; 30(13):2571-2581. http:// dx.doi.org/10.1016/j.biomaterials.2008.12.073. PMid:19157533.

10. Uebersax L, Merkle HP and Meinel L. Insulin-like growth factor I releasing silk fibroin scaffolds induce chondrogenic differentiation of humanmesenchymal stem cells. Journal of Controlled Release. 2008; 127(1):12-21. http://dx.doi. org/10.1016/j.jconrel.2007.11.006. PMid:18280603.

11. Jeon O, Song SJ, Yang HS, Bhang SH, Kang SW, Sung MA, et al. Long-term delivery enhances in vivo osteogenic efficacy of bone morphogenetic protein-2 compared to short-term delivery. Biochemical and Biophysical Research Communications. 2008; 369(2):774-780. http://dx.doi.org/10.1016/j.bbrc.2008.02.099. PMid:18313401. 